psychopraxis. neuropraxis $2021 \cdot 24: 166-171$ https://doi.org/10.1007/s00739-021-00726-z Angenommen: 26. März 2021

Online publiziert:22. April 2021

๑) Der/die Autor(en) 2021

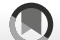

Check for
updates

\section{Dagmar Steinmair ${ }^{1,2} \cdot$ Henriette Löffler-Stastka ${ }^{1}$}

'Abteilung für Psychoanalyse und Psychotherapie, Medizinische Universität Wien, Wien, Österreich

${ }^{2}$ Karl Landsteiner Privatuniversität für Gesundheitswissenschaften, Krems an der Donau, Österreich

\title{
Zusammenhänge zwischen Empathie, therapeutischer Haltung und Wirkeffizienz
}

\section{Einleitung}

Empathie stellt die Grundlage für soziales und moralisches Verhalten dar - häufig wird sie als „einfühlendes Verstehen“ definiert und beinhaltet die Fähigkeit, die Perspektive und Erfahrungswelt anderer wahrnehmen zu können und sie von der eigenen $\mathrm{zu}$ unterscheiden [1].

Empathische Kommunikation beeinflusst Patientenzufriedenheit, Lebensqualität und Schmerzempfinden von Patienten positiv [2-9]. Bisherige Studien zur Kosten- und Wirkeffizienz von Empathie zeigten des Weiteren, dass Vorteile von Empathie evident sind, dass aber eine Erforschung weiterer Zusammenhänge empfehlenswert ist [6, 10]. Empathische Kommunikation reduziert Stress für Patienten und erhöht das Vertrauen in Behandler [11, 12]; Depressivität, Angst, das Risiko für Herzerkrankungen und Mortalität sinken [10-13]. Indirekte Kosten werden gesenkt durch bessere Diagnosen, höhere Therapie-Adhärenz, geringere Hospitalisierungsraten und -dauer [10, $12,14]$. Nicht zuletzt bedeutet ein empathischer Umgang auch für Behandler ein geringeres Burn-out-Risiko sowie eine höhere klinische und rechtliche Sicherheit [15-17]. Im Alltag kann ein Fokus auf empathische Kommunikation einen zeitlich geringgradigen Mehraufwand bedeuten [10]. In psychotherapeutischen Settings kommt ohne Empathie auf Therapeutenseite keine heilsame therapeutische Beziehung zustande, diese ist für einen Therapieerfolg notwendig, aber alleine nicht ausreichend [18]. Empathie wurde in der vergleichenden
Betrachtung von allgemeinen und spezifischen Wirkfaktoren als der Faktor mit der größten Effektstärke für den Therapieerfolg identifiziert [18].

\section{Empathie und Mitgefühl}

Eine Unterscheidung zwischen Mitgefühl und Empathie wird getroffen, um negative Auswirkungen von Empathie hervorzuheben. Empathisches Sich-Einfühlen und Nachempfinden kann dazu führen, dass es schwierig wird, eigene Wünsche und Bedürfnisse von jenen anderer abzugrenzen. Training und Supervision haben bei Psychotherapeuten eine positive Auswirkung auf Lebensqualität und Resilienz gezeigt [19].

\section{》) Erfahrene Therapeuten zeigen eher ein "rational empathisches" Profil}

Eine Analyse der Ausprägung von klinischer Empathie bei Experten $(N=775)$ [20] erhob der Interpersonal Reactivity Index, welcher Perspektivenwechsel (PW), Fantasiefähigkeit (F), empathische Besorgnis (EB) und persönlichen Distress (PD) misst. Die beschriebenen Therapeutenprofile: 1: $23 \%$ „insecure self-absorbed" (unsicher/mit sich selbst beschäftigt: höchster $\mathrm{PD}$, unterdurchschnittlich bei PW, F, EB); $26 \%$ „empathic immersion" (intuitiv, sich identifizierend: höchste EB und überdurchschnittlich bei PW, F); $38 \%$, average “ (durchschnittlich bei PW, F, EB und PD) und $13 \%$ „rational empathic“ (intellektuell verstehend: höchster PW, durchschnittliche EB, ge- ringe $\mathrm{F}$ und geringster $\mathrm{PD}$ ) [20]. Eine multidimensionale Betrachtung empathischer Fähigkeiten zeigt, dass übermäßiges Nachfühlen und Sich-Identifizieren mit dem Patienten zusätzlich zum notwendigen PW dem Therapieerfolg abträglich ist. Damit übereinstimmend: Erfahrene Therapeuten zeigten eher ein „rational empathisches" Profil.

Mit Empathie ist aus psychoanalytischer Sicht ein Set unbewusster und intuitiv ablaufender intra- und interpersonaler Vorgänge gemeint [21]. Philosophische Konzeptualisierungen von Empathie beinhalten neben einer kognitiven Komponente (z.B. Theory of Mind [ToM], inkl. PW) eine phänomenologische, erfahrungsbasierte Komponente (vgl. "embodied empathy“ - z.B. Betonung körperlicher Resonanz und Affektivität) [22-24]. Seelische Belastung, falsche Konsens-Effekte [25] und Missverständnisse führen $\mathrm{zu}$ egozentrischem PW. Ausgehend von eigenen Erfahrungswelten werden jene anderer unzureichend simuliert; dies kann zu emotionaler Überempfindlichkeit führen $[24,26,27]$, welche in sozialen Interaktionen relevant wird.

Im Laufe des Medizinstudiums wurde für Studenten eine abnehmende Empathiefähigkeit in Selbsteinschätzungen dokumentiert [28, 29]. Stress führt $\mathrm{zu}$ Burn-out sowie „compassion fatigue " in helfenden Berufen [30-32]. Ob Interventionen, um kommunikative Fähigkeiten und Empathie zu trainieren effektiv sind, wird kontrovers diskutiert - vor allem besteht noch kein Konsens darüber, welche Methodik hierfür am geeignetsten ist, auch zu Langzeiteffekten fehlen 
noch konsistente Resultate; der in einer Metaanalyse nachgewiesene signifikante Trainingseffekt war für personalisiertes und sehr spezifisches Feedback nachweisbar [27, 33]. Arbeiten zur „wahrgenommenen Verhaltenskontrolle“ gehen davon aus, dass die subjektive Überzeugung und die Erfahrung, Ressourcen zu haben, ein Verhalten auszuführen, dazu beitragen, das Verhalten auch zu realisieren [34]. Eine Erhebung von Fertigkeiten bei Medizinstudenten in Österreich zeigt Verbesserungspotenzial; etwa, dass nur die Hälfte der evaluierten Abteilungen den Studierenden einen konstanten Mentor zugeteilt hatte [35]. Unklarheiten bei Studierenden zu Konzept und Relevanz von Empathie zeigte die Metaanalyse von Costa-Drolon et al. [36]. Patientenrückmeldungen bzgl. der Empathie ihrer Behandler zeigen eine hohe Variabilität [37].

Psychoanalytische Behandlungsmethoden fokussieren auf Training von Containment, Fähigkeit zur Mentalisierung und Affektregulation unter Berücksichtigung von Übertragung und Gegen- übertragungsprozessen [21]. Wohlbefinden und Therapieerfolge stehen in engem Zusammenhang mit zum Teil adaptiven Persönlichkeitseigenschaften wie Resilienz, Optimismus und durch Erfahrung gewonnene Weitsicht [38].

Das beobachtete Verhalten von Bezugspersonen hat Einfluss auf DistressSymptome bei Kindern während medizinischer Eingriffe. In unter Zweijährigen wirkt sich fehlende mitfühlende Nähe während der Maßnahmen negativ aus, während bei älteren Kindern der Distress steigt, wenn negative und bedrohliche Aspekte der Situation ins Zentrum der Aufmerksamkeit gerückt werden [39].

„Weisheit“ beinhaltet, neben kognitivintellektuellen Aspekten, soziale Kompetenz, Emotionsregulation, Ambivalenztoleranz, Reflexionsfähigkeit und Entschlossenheit; sie zeigt sich in der Anwendung von Wissen in Übereinstimmung mit sozialen und persönlichen Interessen [38]. Eine Metaanalyse zeigt, dass prosoziales Verhalten und Emotionsregulation signifikant trainierbar sind [38].
Die willentliche Entscheidung, eine empathische Haltung einzunehmen, scheint nicht ausreichend, um empathisches Verhalten zu erreichen [33, 34, 40], allerdings wird für die kognitive Empathie eine bewusste Steuerbarkeit berichtet. Unbewusste zwischenmenschliche Prozesse sind jedoch unabhängig von Persönlichkeitsmerkmalen und kognitiver ToM. Dies widerspricht Studienergebnissen, welche Menschen mit bestimmten Persönlichkeitsmerkmalen den Willen zum empathischen Handeln, aber nicht die Empathiefähigkeit absprachen [41]. Soziale Emotionen sind jene, welche nicht durch individuelles Erleben evoziert werden, sondern durch die Zugehörigkeit zu einer Gruppe und zu assoziierten Ereignissen unter der Voraussetzung der Ausprägung einer sozialen Identität (z. B. Stolz, Freude, Schuld, Scham, Ingroup-Angst und Wut/ Hass auf eine Outgroup [42]). Entsprechend basiert Training von prosozialem Verhalten auf Beobachtung und Imitation sozialer Emotionen; dies konnte bei schizophrenen Patienten für die kog-

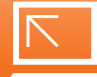

\section{Patientenbroschüre „Angsterkrankungen"}

„Angsterkrankungen. 20 Fragen - 20 Antworten“ erklärt kurz und bündig Grundbegriffe und Alltagsaspekte gut verständlich für den Patienten. So bleibt im Praxisgespräch mehr Zeit für Detailfragen. In Kürze erhältlich!

Die Corona-Pandemie hat sich bei vielen Menschen stark auf die Psyche geschlagen. Soziale Einschränkungen und beruflicher Stress in vielen Formen bereiten Sorgen und führen zu einer Zunahme von Angsterkrankungen. Körperliche Symptome können oft Ausdruck dieser Angst sein und erzeugen zusätzlich Sorgen. Um diesem Kreislauf zu entkommen, geht es zunächst darum, das Problem zu benennen und dann aktiv entgegen zu wirken.

Typische Symptome richtig deuten Patienten mit Angsterkrankungen kommen oft mit vielfältigen körperlichen Beschwerden, für die sich keine organische Ursache finden lässt. Mit der neuen Patientenbroschüre von SpringerMedizin können sich Patienten vorab informieren. Die Broschüre soll helfen, typische Symptome einer Angsterkran- kung zu erkennen und richtig zu deuten. Sie gibt Informationen, wie die Erkrankung in Erscheinung tritt und wie sie sich von anderen Störungen unterscheidet. Weiters erfahren Patienten, welche Behandlungsmöglichkeiten zur Verfügung stehen und welche am erfolgversprechendsten sind. Meist bringt eine Kombination unterschiedlicher Maßnahmen den besten Erfolg. Besonders wichtig: Der Patient wird motiviert, aktiv an seiner Behandlung mitzuarbeiten.

$>$ Ab Ende Juni verfügbar

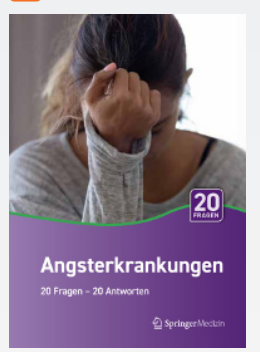

Die Patientenbroschüre „Angsterkrankungen. 20 Fragen - 20 Antworten“ von Springer Medizin mit Unterstützung von Schwabe Austria GmbH steht ab Ende Juni kostenlos für Ihre Patienten zur Verfügung.

Bestellung unter: ad@schwabe.at 
nitive als auch für die affektive ToM gezeigt werden [43]. Eine Verbesserung der „social cognition“ durch ein Training der Mentalisierungsfähigkeit bei Ärzten und Therapeuten einer psychiatrischen Abteilung konnte mittels videobasiertem Assessment nachgewiesen werden (Movie for the Assessment of Social Cognition) [27, 44].

\section{Kasuistik}

Folgendes Fallbeispiel dient dazu, das Konzept der mentalisierten Affektivität und Defizite in dieser Fähigkeit zu beleuchten. Am Beispiel der Schilderung des Falls A. sollen Herausforderungen einer ambulanten Patientenbetreuung aufgezeigt werden. Frau A., Anfang dreißig, abwechselnd als paranoid, negativistisch und „Borderline“ beschrieben, war nach stationärer Behandlung nach einem Suizidversuch in Psychotherapie. Auffallend waren eine Selbstvernachlässigung und bösartige, aber amüsante Kommentare über andere sowie konfuse Assoziationen. Ein Misshandlungsgeschehen in Frau A.s Jugend war anamnestisch bekannt. Die Arbeit hatte sie verloren, die Umgebung und die Mitmenschen ließ sie durch ihr wegstoßend, zerstörerisches und zum Teil beschuldigendes Verhalten leiden. Im Zuge der Therapieplanung verhielt sie sich selbstdestruktiv - ausgelöst durch eine Unfähigkeit, Affekte zu regulieren. Zu Beginn der Therapie waren besonders die Fehlinterpretation des Verhaltens anderer (z.B. Unterstellung böser Absicht, leichte Kränkbarkeit) sowie der überwiegend negative Affekt (v. a. Wut) und eine desorganisierte Bindung mit Isolation auffallend. Sie vernachlässigte ihren Gesundheitszustand, heizte die Wohnung nicht und wurde wegen einer Pneumonie internistisch stationär behandelt. Während Frau A. eine Begegnung mit dem Konsiliarpsychiater schilderte, in welcher sie auf die Frage: „Denken Sie manchmal daran, sich selbst etwas anzutun?“, geantwortet hatte: „Nein, ich denke daran, das Spital zu klagen!“ nahm die Therapeutin eine Überflutung der eigenen Psyche mit Frau A.s Gefühlen wahr. Frau A. musste die Standardfrage als Affront interpretiert haben. Der Aufbau einer therapeutischen Beziehung ge- lang - wobei die Therapeutin Schwierigkeiten mit der eigenen Affektregulierung im Sinne einer Gegenübertragungsreaktion feststellte. Frau A.s teils mit Humor abgewehrte Aggression bot der Therapeutin eine Möglichkeit, Kontakt herzustellen. In der Therapie äußerte sie basierend auf der Beobachtung einer aus ihrer Sicht müde wirkenden Therapeutin: „Sehen Sie sich doch mal an! Haben Sie denn überhaupt keine Ahnung ... Sie könnten sterben, wenn Sie so ... mein Blick könnte sie töten“. Die Äußerung legt nahe, dass Frau A. die scheinbare „Müdigkeit“ der Therapeutin als auch für sie bedrohlich oder als Provokation empfand. Die Feststellung beendet sie mit der Empfehlung, die Therapeutin solle sich einen Kaffee holen. Gewissermaßen soll die Bedrohung der Therapeutin durch ihren Blick wiedergutgemacht werden. Die fürsorgliche aber triumphierende Äußerung kann als konstruktiver Umgang mit Aggression und Libido betrachtet werden. Das notwendige Containment destruktiver Impulse und Affekte muss immer wieder geleistet und in Worte gefasst werden.

\section{Diskussion und Ausblick}

Interesse an der eigenen inneren Welt und jener des Patienten ist eine Voraussetzung für eine lebendige Erfahrung in der Therapie, welche sich vom theoretischen Verstehen einer Situation unterscheidet. Identifizierung, Modulierung und Äußerung von Affekten bedingen empathisches Verstehen und Verhalten. Therapeutisch wird an der Einstellung des Patienten zu seiner Affektivität gearbeitet. Im Falle einer projektiven Identifizierung, wie sie im oben beschriebenen Beispiel dargestellt ist, wird die Psyche des Therapeuten durch unerträgliche und widersprüchliche Affekte des Patienten überflutet, die durch die Patientin nur zum Teil wahrnehmbar und nicht integrierbar blieben (vgl. auch [45]). Ohne die Möglichkeit, Gedanken als Vorstellungen in einem Möglichkeitsraum zu repräsentieren, ist der Mensch zum (repetitiven) Agieren prädisponiert. Die Entfaltung des Selbst als auch der Aufbau einer Beziehung zu anderen hängen entscheidend davon $\mathrm{ab}, \mathrm{ob}$ die eigene innere psychopraxis. neuropraxis $2021 \cdot 24$ :

166-171

https://doi.org/10.1007/s00739-021-00726-z

(c) Der/die Autor(en) 2021

\section{Steinmair · H. Löffler-Stastka}

\section{Zusammenhänge zwischen Empathie, therapeutischer Haltung und Wirkeffizienz}

\section{Zusammenfassung}

Empathisch sein heißt, fühlen und verstehen können, was andere fühlen. Vermuten zu können, was das Gegenüber fühlt, denkt und wünscht, beruht auf der Fähigkeit, eigene Gefühle und Gedanken als getrennt von jenen anderer wahrnehmen und regulieren zu können. Definierte Therapieerfolge mit adäquatem Aufwand erreichen zu können, verlangt ein Fokussieren auf Wesentliches und Wichtiges. Die Empathie ist im Bereich der Psychotherapie jener Faktor, für den für sich genommen die höchste Effektstärke nachgewiesen werden konnte. Empathietraining ermöglicht eine bessere soziale Performance. Im Falle von Defiziten in sozialer Kompetenz ist störungsunabhängig ein besonders hoher Leidensdruck nachweisbar.

\section{Schlüsselwörter}

Verkörperte Empathie · Theorie des Geistes . Soziale Emotionen · Unbewusstes · Soziale Kognition

\section{Connections Between Empathy, Therapeutic Attitude and Efficacy}

\section{Abstract}

Empathy means feeling and understanding what others are feeling. Being able to imagine what the other person feels, thinks, and wishes is based on the ability to sense and regulate one's own feelings and thoughts separately from those of others. Achieving desired therapy outcomes with adequate effort requires focussing on what is essential and important. In the field of psychotherapy, empathy is the one factor with the most significant effect size. Empathy training enables better social performance. In the case of social skills deficits, a particularly high level of distress is demonstrated, independent of the mental health condition.

\section{Keywords}

Embodied empathy · Theory of Mind · Social emotions · Unconscious · Social cognition 
Welt als solche abgrenzbar, kohärent und beherrschbar ist oder ob sie mit jener des Gegenübers verschwimmt.

》) Identifizierung, Modulierung
und Äußerung von Affekten
bedingen empathisches
Verstehen und Verhalten

Angehende Mediziner sehen empathische Fähigkeiten als wichtig an, allerdings variiert diese Haltung je nach gewählter fachärztlicher Spezialisierung [46]. Bei Medizinstudenten wurden Alter, Geschlecht, Land, Zweck und Art der Intervention/Messung und das Vorhandensein einer Trainingsmöglichkeit als Moderatoren der Empathie erhoben [47].

Perspektivenwechsel lassen sich auch in einem „virtual reality training“" signifikant verbessern - allerdings ist die erzielte Verbesserung nicht signifikant für Empathie [48]. Bei der Erstellung von E-Learning-Fällen gilt es neben der Vermittlung von theoretischem Wissen (vgl. deklaratives Gedächtnis), auch die klinische Perspektive erfahrbar zu machen [49], wobei die Vermittlung von Skills (vgl. prozedurales Gedächtnis) und affektiver ToM via E-Learning limitiert ist. Rollenspiele anhand von standardisierten Patienten stellen eine wichtige Möglichkeit dar [50]. Rollenspiele affektiver Störungen, welche besonders hohe Anforderungen an die Vermittlung affektiver ToM stellen, werden von Experten als weniger realitätsnah beurteilt. Achtsamkeit konnte via Smartphone-App signifikant gebessert werden - passend zur besseren Vermittelbarkeit der kognitiven ToM [51].

Ein konstruktiver Umgang mit Empathie muss geübt werden - um negative direkte und indirekte Folgen abzuwenden [2, 8, 21, 30-32]: Mentorship-Programme, Fairness in der Verteilung der Arbeitslast, Anerkennung der Leistung und Rückhalt im Team, Maßnahmen, welche die Resilienz positiv beeinflussen und Möglichkeiten für Training und Weiterentwicklung wirken entgegen.

\section{Fazit für die Praxis}

- Empathische Therapeuten lassen diese Fähigkeit in ihr therapeutisches Handeln einfließen und zeigen Wirkeffizienz.

- Prosoziales Verhalten wird bereits im Kindesalter in wiederholten Interaktionen mit den wichtigen Bezugspersonen vermittelt und ist an die Ausbildung einer sozialen Identität gekoppelt. Die Vermittlung von prosozialen und proempathischen klinischen Skills ist evidenzbasiert und effektiv, selbst wenn sie zu einem späteren Zeitpunkt im Leben erfolgt.

- Ein Empathietraining ist effektiv, wenn es auf die Vermittlung von Fähigkeiten wie Containment, Mentalisierungsfähigkeit und Emotionsregulation fokussiert, und darauf, eigene Erfahrungswelten nicht auf das Gegenüber zu übertragen (vgl. Abstinenz, Übertragung/ Gegenübertragung).

\begin{tabular}{l} 
Univ. Prof. Dr. med. univ. \\
Henriette Löffler-Stastka \\
Abteilung für Psychoanalyse \\
und Psychotherapie, \\
$\begin{array}{l}\text { Medizinische Universität } \\
\text { Wien } \\
\text { Währinger Gürtel 18-20, } \\
1090 \text { Wien, Österreich } \\
\text { henriette.loeffler-stastka@ } \\
\text { meduniwien.ac.at }\end{array}$ \\
\hline
\end{tabular}

Funding. Open access funding provided by Medical University of Vienna.

\section{Einhaltung ethischer Richtlinien}

Interessenkonflikt. D. Steinmair und H. LöfflerStastka geben an, dass kein Interessenkonflikt besteht.

Für diesen Beitrag wurden von den Autoren keine Studien an Menschen oder Tieren durchgeführt. Für die aufgeführten Studien gelten die jeweils dort angegebenen ethischen Richtlinien.

Open Access. Dieser Artikel wird unter der Creative Commons Namensnennung 4.0 International Lizenz veröffentlicht, welche die Nutzung, Vervielfältigung Bearbeitung, Verbreitung und Wiedergabe in jeglichem Medium und Format erlaubt, sofern Sie den/die ursprünglichen Autor(en) und die Quelle ordnungsgemäß nennen, einen Link zur Creative Commons Lizenz beifügen und angeben, ob Änderungen vorgenommen wurden.

Die in diesem Artikel enthaltenen Bilder und sonstiges Drittmaterial unterliegen ebenfalls der genannten Creative Commons Lizenz, sofern sich aus der Abbildungslegende nichts anderes ergibt. Sofern das betreffende Material nicht unter der genannten Creative Commons Lizenz steht und die betreffende Handlung nicht nach gesetzlichen Vorschriften erlaubt ist, ist für die oben aufgeführten Weiterverwendungen des Materials die Einwilligung des jeweiligen Rechteinhabers einzuholen.

Weitere Details zur Lizenz entnehmen Sie bitte der Lizenzinformation auf http://creativecommons.org/ licenses/by/4.0/deed.de.

\section{Literatur}

1. Stangl W (2021) Stichwort: „Empathie“. Online Lexikon für Psychologie und Pädagogik. https:// lexikon.stangl.eu/1095/empathie. Zugegriffen: 06.03.2021

2. Howick J, Moscrop A, Mebius A, Fanshawe TR, Lewith G, Bishop FL, Mistiaen P, Roberts NW, Dieninyte E, Hu XY, Aveyard P, Onakpoya IJ (2018) Effects of empathic and positive communication in healthcare consultations: a systematic review and meta-analysis. J R Soc Med 111(7):240-252. https://doi.org/10.1177/0141076818769477

3. Chassany O, Boureau F, Liard F, Bertin P, Serrie A, Ferran P, Keddad K, Jolivet-Landreau I, Marchand S (2006) Effects of training on general practitioners' management of pain in osteoarthritis: a randomized multicenter study. J Rheumatol 33(9):1827-1834

4. White $P$, Bishop FL, Prescott $P$, Scott $C$, Little $P$, Lewith G (2012) Practice, practitioner, or placebo? A multifactorial, mixed-methods randomized controlled trial of acupuncture. Pain 153(2):455-462. https://doi.org/10.1016/j.pain.2011.11.007

5. Kaptchuk TJ, Kelley JM, Conboy LA, Davis RB, Kerr CE, Jacobson EE, Kirsch I, Schyner RN, Nam BH, Nguyen LT, Park M, Rivers AL, McManus C, Kokkotou E, Drossman DA, Goldman P, Lembo AJ (2008) Components of placebo effect: randomised controlled trial in patients with irritable bowel syndrome. BMJ 336(7651):999-1003. https://doi. org/10.1136/bmj.39524.439618.25

6. Mercer SW, Fitzpatrick B, Guthrie B, Fenwick E, Grieve E, Lawson K, Boyer N, McConnachie A, Lloyd SM, O'Brien R, Watt GC, Wyke S (2016) The CARE plus study - a whole-system intervention to improve quality of life of primary care patients with multimorbidity in areas of high socioeconomic deprivation: exploratory cluster randomised controlled trial and cost-utility analysis. BMC Med 14(1):88. https://doi.org/10.1186/s12916-0160634-2

7. Fujimori M, Shirai Y, Asai M, Kubota K, Katsumata N, Uchitomi Y (2014) Effect of communication skills training program for oncologists based on patient preferences for communication when receiving bad news: a randomized controlled trial. J Clin Oncol32(20):2166-2172.https://doi.org/10.1200/ JCO.2013.51.2756

8. Vangronsveld KL, Linton SJ (2012) The effect of validating and invalidating communication on satisfaction, pain and affect in nurses suffering from low back pain during a semi-structured interview. Eur J Pain 16(2):239-246. https://doi. org/10.1016/j.ejpain.2011.07.009 


\section{Psychiatrie}

9. Soltner C, Giquello JA, Monrigal-Martin C Beydon L (2011) Continuous care and empathic anaesthesiologist attitude in the preoperative period: impact on patient anxiety and satisfaction. $\mathrm{Br} J$ Anaesth 106(5):680-686. https://doi.org/10. 1093/bja/aer034

10. Howick J, Mittoo S, Abel L, Halpern J, Mercer SW (2020) A price tag on clinical empathy? Factors influencing its cost-effectiveness. J R Soc Med 113(10):389-393. https://doi.org/10.1177/ 0141076820945272

11. Weiss R, Vittinghoff E, Fang MC, Cimino J, Chasteen KA, Arnold RM, Auerbach AD, Anderson WG (2017) Associations of physician empathy with patient anxiety and ratings of communication in hospital admission encounters. J Hosp Med 12(10):805-810. https://doi.org/10.12788/jhm. 2828

12. Riess $H$ (2017) The science of empathy. J Patient Exp 4(2):74-77. https://doi.org/10.1177/ 2374373517699267

13. Benson H, Greenwood MM, KlemchukH (1975) The relaxation response: psychophysiologic aspects and clinical applications. Int J Psychiatry Med 6(1-2):87-98. https://doi.org/10.2190/376WE4MT-QM6Q-HOUM

14. Butler CC, Pill R, Stott NC (1998) Qualitative study of patients' perceptions of doctors' advice to quit smoking: implications for opportunistic health promotion. BMJ 316(7148):1878-1881. https:// doi.org/10.1136/bmj.316.7148.1878

15. Moore PJ, Adler NE, Robertson PA (2000) Medical malpractice: the effect of doctor-patient relations on medical patient perceptions and malpractice intentions. West J Med 173(4):244-250. https:// doi.org/10.1136/ewjm.173.4.244

16. Sturzu L, Lala A, Bisch M, Guitter M, Dobre D, Schwan R (2019) Empathy and burnout-a cross-sectional study among mental healthcare providers in France. JMedLife 12(1):21-29. https:// doi.org/10.25122/jml-2018-0050

17. Doyle CR, Akhtar J, Mrvos R, Krenzelok EP (2004) Mass sociogenic illness-real and imaginary. Vet Hum Toxicol 46(2):93-95

18. Wampold BE (2015) How important are the common factors in psychotherapy? An update. World J Psychiatry 14(3):270-277. https://doi.org/ 10.1002/wps.20238

19. Laverdière O, Ogrodniczuk JS, Kealy D (2019) Clinicians' empathy and professional quality of life. J Nerv Ment Dis 207(2):49-52. https://doi.org/10. 1097/NMD.0000000000000927

20. Laverdière O, Kealy D,OgrodniczukJS, Descôteaux (2019) Got empathy? A latent profile analysis of psychotherapists' empathic abilities. Psychother Psychosom 88(1):41-42. https://doi.org/10.1159/ 000494141

21. Löffler-Stastka H, Datz F, Parth K, Preusche I, Bukowski X, Seidman C (2017) Empathy in psychoanalysis and medical education - what can we learn from each other? BMC Med Educ 17:74. https://doi.org/10.1186/s12909-017-0907-2

22. Schmidsberger F, Löffler-Stastka H (2018) Empathy is proprioceptive: the bodily fundament of empathy-a philosophical contribution to medical education. BMC Med Educ 18:69. https://doi.org/ 10.1186/s12909-018-1161-y

23. Fuchs T (2016) Intercorporeality and Interaffectivity. In: Meyer C, Streeck J, Jordan S (Hrsg) Intercorporeality: emerging socialities in interaction. Oxford University Press, Oxford https://doi.org/ 10.1093/acprof:oso/9780190210465.003.0001
24. Slaby J (2014) Empathy's blind spot. Med Health Care Philos 17(2):249-258. https://doi.org/10. 1007/s11019-014-9543-3

25. Ross L, Greene D, House P (1977) The "false consensus effect": an egocentric bias in social perception and attribution processes. J Exp Soc Psychol 13(3):279-301

26. Coplan A, Goldie P (2011) Empathy: philosophical and psychological perspectives. Oxford University Press, Oxford, S431

27. Steinmair D, Horn R, Richter F, Wong G, LöfflerStastka H (2021) Mind reading improvements by mentalization based therapy training. Bull Menninger Clin 85(1):59-83

28. SeitzT,GruberB,Preuschel,Löffler-Stastka H(2017) Rückgang von Empathie der Medizinstudierenden im Laufe des Studiums - was ist die Ursache? ZPsychosom Med Psychother 63(1):20-39. https:// doi.org/10.13109/zptm.2017.63.1.20

29. Spatoula V, PanagopoulouE,MontgomeryA(2019) Does empathy change during undergraduate medical education? - a meta-analysis. Med Teach 41(8):895-904. https://doi.org/10.1080/ 0142159X.2019.1584275

30. Zhang YY, Zhang C, Han XR, Li W, Wang YL (2018) Determinants of compassion satisfaction, compassion fatigue and burn out in nursing: a correlative meta-analysis. Medicine 97(26):e11086. https:// doi.org/10.1097/MD.0000000000011086

31. Zhang YY, Han WL, Qin W, Yin HX, Zhang CF, Kong C, Wang YL (2018) Extent of compassion satisfaction, compassion fatigue and burnout in nursing: a meta-analysis. J Nurs Manag 26(7):810-819. https://doi.org/10.1111/jonm.12589

32. Sinclair S, Raffin-Bouchal S, Venturato L, Mijovic Kondejewski J, Smith-MacDonald L (2017) Compassion fatigue: a meta-narrative review of the healthcare literature. Int J Nurs Stud 69:9-24. https://doi.org/10.1016/j.jinurstu.2017.01.003

33. Gruber MB (2015) Klinische Empathie - ein altes Werkzeug neu entdeckt. Medizinstudierende wollen sich empathisch verhalten - was beeinflusst sie? Eine Anwendung der Theorie des geplanten Verhaltens. http://othes.univie.ac.at/ 39971/1/2015-10-08 0603573.pdf. Zugegriffen: 06.03.2021

34. Ajzen I (2011) The theory of planned behaviour: reactions and reflections. Psycho Health 26(9):1113-1127. https://doi.org/10.1080/ 08870446.2011 .613995

35. Löffler-Stastka H, Seitz T, Raschauer B, Längle AS (2019) Competency in medical history taking: the training physicians view. Wien Klin Wochenschr 131:17-22. https://doi.org/10.1007/s00508-0181431-z

36. Costa-Drolon E, Verneuil L, Manolios E, RevahLevy A, Sibeoni J (2021) Medical students' perspectives on empathy: a systematic review and metasynthesis. Acad Med 96(1):142-154. https:// doi.org/10.1097/ACM.0000000000003655

37. Howick J, Steinkopf L, Ulyte A, Roberts N, Meissner K (2017) How empathic is your healthcare practitioner? A systematic review and metaanalysis of patient surveys. BMC Med Educ 17:136. https://doi.org/10.1186/s12909-017-0967-3

38. Lee EE, Bangen KJ, Avanzino JA, Hou B, Ramsey M Eglit G, Liu J, Tu XM, Paulus M, Jeste DV (2020) Outcomes of randomized clinical trials of interventions to enhance social, emotional, and spiritual components of wisdom: a systematic review and metaanalysis. JAMA Psychiatry 77(9):925-935. https:// doi.org/10.1001/jamapsychiatry.2020.0821

39. Sobol-Kwapińska M, Sobol M, Woźnica-Niesobs ka E (2020) Parental behavior and child distress and pain during pediatric medical procedures: systematic review and meta-analysis. Health Psychol 39(7):558-572. https://doi.org/10.1037/ hea0000864

40. Datz F, Wong G, Löffler-Stastka H (2019) Interpretation and working through contemptuous facial micro-expressions benefits the patienttherapist relationship. Int J Environ Res Public Health 16(24):4901. https://doi.org/10.3390/ ijerph16244901

41. Kajonius PJ, Björkman T (2020) Individuals with dark traits have the ability but not the disposition to empathize. Pers Individ Dif 155:109716. https:// doi.org/10.1016/j.paid.2019.109716

42. Rees J (2021) Emotionen, gruppenbasierte im Dorsch Lexikon der Psychologie. https:// dorsch.hogrefe.com/stichwort/emotionengruppenbasierte. Zugegriffen: 2. März 2021

43. Yeh P-Y, Yu L, Guo N-W, Lin W-C, Wu C-K (2019) Observation and imitation of social emotions are essential for improving cognitive and affective theory of mind in schizophrenia: a meta-analysis. J Nerv Ment Dis 207(6):474-481. https://doi.org/ 10.1097/NMD.0000000000000996

44. Dziobek I, Fleck S, Kalbe E, Rogers K, Hassenstab J, Brand M, Kessler J, Woike JK, Wolf OT, Convit A (2006) Introducing MASC: a movie for the assessment of social cognition. J Autism Dev Disord 36(5):623-636. https://doi.org/10.1007/ s10803-006-0107-0

45. Fonagy P, Gergely G, Jurist EL, Target M (2004) Affektregulierung, Mentalisierung und die Entwicklung des Selbst. Klett-Cotta, Stuttgart, S 442-446

46. Zervos K (2019) Anamnesekompetenz bei Medizinstudierenden - die Sicht der Ausbildungsverantwortlichen. Medizinische Universität Wien, Wien

47. Fragkos KC, Crampton P (2020) The effectiveness of teaching clinical empathy to medical students: a systematic review and meta-analysis of randomized controlled trials. Acad Med 95(6):947-957. https://doi.org/10.1097/ACM. 0000000000003058

48. Ventura S, Badenes-Ribera L, Herrero R, Cebolla A Galiana L, Baños R (2020) Virtual reality as a medium to elicit empathy: a meta-analysis. Cyberpsychol Behav Soc Netw 23(10):667-676. https://doi.org/10.1089/cyber.2019.0681

49. Turk BR, Krexner R, Otto F, Wrba T, Löffler-Stastka $H$ (2015) Not the ghost in the machine: transforming patient data into E-learning cases within a casebased blended learning framework for medical education. Procedia Soc Behav Sci 186:713-725. https://doi.org/10.1016/j.sbspro.2015.04.106

50. Himmelbauer M, Seitz T, Seidman C, LöfflerStastka H (2018) Standardized patients in psychiatry-the best way to learn clinical skills? BMCMed Educ 18(1):72. https://doi.org/10.1186/s12909018-1184-4

51. Linardon J (2020) Can acceptance, mindfulness, and self-compassion be learned by smartphone apps? A systematic and meta-analytic review of randomized controlled trials. Behav Ther 51(4):646-658. https://doi.org/10.1016/j.beth. 2019.10.002

Hinweis des Verlags. Der Verlag bleibt in Hinblick auf geografische Zuordnungen und Gebietsbezeichnungen in veröffentlichten Karten und Institutsadressen neutral. 
Hier steht eine Anzeige.

\section{曾 Springer}

